\title{
Reoxygenation induces reactive oxygen species production and ferroptosis in renal tubular epithelial cells by activating aryl hydrocarbon receptor
}

\author{
THEODOROS ELEFTHERIADIS, GEORGIOS PISSAS, GEORGIOS FILIPPIDIS, \\ VASSILIOS LIAKOPOULOS and IOANNIS STEFANIDIS
}

Department of Nephrology, Faculty of Medicine, University of Thessaly, 41110 Larissa, Greece

Received August 10, 2020; Accepted October 8, 2020

DOI: $10.3892 / \mathrm{mmr} .2020 .11679$

\begin{abstract}
During the reperfusion phase of ischemia-reperfusion injury, reactive oxygen species (ROS) production aggravates the course of many diseases, including acute kidney injury. Among the various enzymes implicated in ROS production are the enzymes of the cytochromes P450 superfamily (CYPs). Since arylhydrocarbon receptor (AhR) controls the expression of certain CYPs, the involvement of this pathway was evaluated in reperfusion injury. Because AhR may interact with the nuclear factor erythroid 2-related factor 2 (Nrf2) and the hypoxia-inducible factor- $1 \alpha$ (HIF-1 $\alpha)$, whether such an interaction takes place and affects reperfusion injury was also assessed. Proximal renal proximal tubular epithelial cells were subjected to anoxia and subsequent reoxygenation. At the onset of reoxygenation, the AhR inhibitor CH223191, the HIF-1 $\alpha$ activator roxadustat, or the ferroptosis inhibitor $\alpha$-tocopherol were used. The activity of AhR, Nrf2, HIF-1 $\alpha$, and their transcriptional targets were assessed with western blotting. ROS production, lipid peroxidation and cell death were measured with colorimetric assays or cell imaging. Reoxygenation induced ROS production, lipid peroxidation and cell ferroptosis, whereas $\mathrm{CH} 223191$ prevented all. Roxadustat did not affect the above parameters. Reoxygenation activated AhR and increased CYP1A1, while CH223191 prevented both. Reoxygenation with or without $\mathrm{CH} 223191$ did not alter $\mathrm{Nrf} 2$ or HIF-1 $\alpha$ activity. Thus, AhR is activated during reoxygenation and induces ROS production, lipid peroxidation and ferroptotic cell death. These detrimental effects may be mediated by AhR-induced CYP overexpression, while the Nrf2 or the HIF-1 $\alpha$ pathways remain unaffected. Accordingly, the AhR
\end{abstract}

Correspondence to: Dr Theodoros Eleftheriadis, Department of Nephrology, Faculty of Medicine, University of Thessaly, Mezourlo Hill, Biopolis 1, Building A, 41110 Larissa, Greece

E-mail: teleftheriadis@yahoo.com

Key words: ischemia-reperfusion injury, arylhydrocarbon receptor, cytochrome $\mathrm{p} 450$, nuclear factor erythroid 2-related factor 2, hypoxia-inducible factor, ferroptosis pathway may represent a promising therapeutic target for the prevention of reperfusion injury.

\section{Introduction}

Ischemia-reperfusion (I-R) injury plays an important role in the pathogenesis of several diseases, such as stroke, coronary heart disease and multiorgan failure (1-3). Due to the high metabolic demands of renal tubular cells, the kidney is exceptionally vulnerable to I-R, and I-R injury is the most common cause of acute kidney injury (4). I-R injury consists of two consecutive, but distinct, stages. During ischemia, cell energy collapse induces cell death, whereas during reperfusion, cell death results from increased reactive oxygen species (ROS) production (1-3). Regarding renal tubular epithelial cells, a study demonstrated that under anoxia, cells die through apoptosis, whereas reoxygenation induces lipid peroxidation-induced cell death, also known as ferroptosis $(5,6)$. The latter has also been detected in isolated mouse renal tubules, confirming the different pathophysiology of ischemia- and reperfusion-induced cell injury (7).

Ferroptosis is a type of regulated iron-dependent cell necrosis mainly caused by increased redox imbalance and characterized by extensive lipid peroxidation and, eventually, severe cell membrane dysfunction (8). Unlike uncontrolled cell necrosis, which occurs acutely after a severe physical, chemical, or mechanical insult, ferroptosis and other types of regulated cell necrosis involve a genetically encoded machinery, occur in a delayed manner, and could be considered as a part of an adaptive response that unsuccessfully attempts to restore cellular homeostasis (8). Thus, contrary to uncontrolled necrosis, for ferroptosis, pharmacological intervention is possible (8). Apoptosis is another type of programmed cell death, which differs in many ways from the various types of regulated cell necrosis. A notable difference is that during apoptosis, cell membrane rupture does not take place, and the release of cytoplasmic content and the ensuing inflammation are avoided (8).

When metabolizing their substrates, the enzymes of the cytochrome P450 superfamily (CYP) produce ROS (9). By oxidizing their substrates, often drugs or xenobiotics, CYPs increase their polarity, aiding to their excretion. 
Substrate oxidation by CYPs is achieved by a six-step reaction during which heme-thiolate iron fluctuates between ferric $\left(\mathrm{Fe}^{+3}\right)$ and ferrous $\left(\mathrm{Fe}^{+2}\right)$ form, interacts with oxygen, and eventually oxidize the substrate. The general reaction is $\mathrm{RH}+2 \mathrm{H}^{+}+2 \mathrm{e}^{-} \rightarrow \mathrm{ROH}+\mathrm{H}_{2} \mathrm{O}$. However, two shunts exist in the six-step CYP catalytic pathway. The first takes place in step 3 of the reaction and gives rise to superoxide. The second occurs in step 4 of the reaction and releases hydrogen peroxide $(9,10)$. In addition to the above mechanism of CYP-induced ROS production, a supplementary mechanism has also been identified. CYPs metabolize arachidonic acid to produce 20-hydroxyeicosatetraenoic acid (20-HETE). The latter increases NADPH oxidase activity resulting in further ROS production $(11,12)$. The role of CYPs in producing ROS and eventually cell death has been confirmed in experimental models of heart and liver I-R injury (13-17).

Certain CYPs are under the transcriptional control of the aryl hydrocarbon receptor (AhR). Non-ligand bound AhR is retained in the cytoplasm as an inactive complex with various factors, which also protect AhR from proteasomal degradation. When activated by exogenous or endogenous ligands, AhR is released, translocates into the nucleus, forms a complex with HIF-1 $\beta$, and transcribes the CYPs CYP1A1, CYP1A2, and CYP1B1 $(18,19)$. Interestingly, activated AhR becomes vulnerable to proteasomal degradation, leading to decreased AhR cellular levels (20-24).

Several studies have suggested that AhR-induced CYP overexpression results in ROS production (25-29). For instance, in human aortic endothelial cells, 2,3,7,8-tetrachlorodibenzo-p-dioxin activates $\mathrm{AhR}$ and induces CYP1A1 expression and ROS production. In this model, small interfering RNA targeting of AhR or CYP1A1 decreased ROS production significantly, confirming that CYPs could mediate AhR-induced ROS production (27). However, the role of this pathway during reperfusion remains unclear.

Although AhR induces oxidative stress by increasing CYPs, it also promotes the expression of nuclear factor erythroid 2-related factor 2 (Nrf2) (30-32). Nrf2 is kept in the cytoplasm by kelch-like ECH-associated protein 1 (KEAP1) and cullin-3. Cullin-3 ubiquitinates Nrf2 leading it to proteasomal degradation. Under increased ROS levels, the conformation of KEAP1 changes releasing $\mathrm{Nrf} 2$, which translocates into the nucleus and transcribes genes encoding antioxidant and anti-ferroptotic proteins, as well as proteins required for detoxification $(33,34)$. At first glance, AhR-induced Nrf2 might attenuate the oxidative stress produced by AhR-induced CYPs (30-32). A study has demonstrated that the kynurenine-AhR pathway mediates brain damage after experimental stroke, a condition that involves both phases of I-R injury (35). Moreover, inhibition of AhR through downregulation of CYPs and ROS protects newborn rat lungs from hyperoxia (25). Thus, evidence indicates that in the context of oxidative stress, the balance between AhR-induced CYPs and AhR-induced Nrf2 tilts towards the former. Besides the detection of a possible detrimental effect of AhR activation in I-R injury or hyperoxia, the aforementioned studies did not assess Nrf2, nor the effect the reperfusion per se. Thus, the precise interaction between AhR and Nrf2 during the reperfusion stage of I-R-injury has not been evaluated.
Another transcription factor that is inevitably affected by I-R is hypoxia-inducible factor- $1 \alpha$ (HIF- $1 \alpha)$. Under normoxia, HIF-prolyl-hydroxylases are active, leading to HIF-1 $\alpha$ proteasomal degradation. Under anoxia, hydroxylation does not take place, and HIF-1 $\alpha$ is released from the Von Hippel-Lindau E3 ubiquitin ligase, escaping proteasomal degradation. The accumulated HIF-1 $\alpha$ forms a heterodimer with HIF-1 $\beta$ and transcribes many genes necessary for cellular adaptation to anoxia $(36,37)$. However, activated AhR also forms a heterodimer with HIF-1 $\beta(33,34)$. In certain experimental models of AhR activation, the competition between AhR and HIF-1 $\alpha$ for HIF-1 $\beta$ results in reduced HIF-1 $\alpha$ transcriptional activity, and vice versa (38-40). However, this interaction between AhR and HIF-1 $\alpha$ has not been evaluated in models of I-R injury.

Most studies have evaluated I-R-injury as a one-step process (1-3), yet I-R consists of two consecutive but different stages, the first of ischemia, and the second of reperfusion (1-3). The present study aimed to evaluate the role of AhR during the reoxygenation phase, and more precisely, its effect on ROS producing CYPs, Nrf2, HIF-1 $\alpha$ and cell survival. A cell culture model was established, in which primary murine renal proximal tubular epithelial cells (RPTECs) were subjected to anoxia, a condition that imitates ischemia, and then to reoxygenation and replenishment with fresh culture medium, a condition that mimics reperfusion. RPTECs are particularly vulnerable to I-R injury (4).

To evaluate the role of AhR in reoxygenation-induced cell death the specific AhR inhibitor $\mathrm{CH} 223191$ was applied at the onset of reoxygenation (41). The HIF-prolyl-hydroxylase inhibitor roxadustat was used to assess the possible role of interaction between AhR and HIF-1 $\alpha$ (42). Finally, the ferroptosis inhibitor $\alpha$-tocopherol (43) was applied at the onset of reoxygenation to examine the effect of AhR on ferroptotic cell death specifically.

\section{Materials and methods}

Cell culture and treatment. Primary C57BL/6 murine RPTECs (cat. no. C57-6015) and Complete Epithelial Cell Medium kit (cat. no. M6621) were purchased from Cell Biologics. Primary cells were used instead of genetically altered cell lines in order to obtain reliable results. Second-passage RPTECs were cultured in 96-well plates $\left(10^{4}\right.$ cells/well) or 6 -well plates $\left(3 \times 10^{5}\right.$ cells/well $)$ at $37^{\circ} \mathrm{C}$. In order to reproduce ischemic conditions, cells were placed in a GasPak ${ }^{\mathrm{TM}}$ EZ Anaerobe Container System with Indicator (cat. no. 26001; BD Biosciences), which reduces the concentration of oxygen to $<1 \%$. To replicate reperfusion, cells were then removed from the Anaerobe Container System and washed with PBS. Fresh culture medium was added, and cells were cultured in a humidified atmosphere containing $5 \% \mathrm{CO}_{2}$.

The selection of appropriate culture time points was based on a previous study demonstrating that primary mouse RPTECs died after $48 \mathrm{~h}$ of anoxia or $4 \mathrm{~h}$ of reoxygenation (5). The latter was also confirmed in the present study. Thus, in this study, cells were subjected to anoxia for $24 \mathrm{~h}$ and subsequently to reoxygenation for $2 \mathrm{~h}$, as after these time points, cell death precludes any further reliable analysis. 
At the onset of the reoxygenation period, $3 \mu \mathrm{M}$ of the $\mathrm{AhR}$ inhibitor CH223191 (cat. no. C8124; Sigma-Aldrich; Merck $\mathrm{KGaA}$ ) or $100 \mu \mathrm{M}$ of the ferroptosis inhibitor $\alpha$-tocopherol (cat. no. T3251; Sigma-Aldrich; Merck KGaA) or the HIF-1 $\alpha$ activator roxadustat at a concentration of $10 \mu \mathrm{g} / \mathrm{ml}$ (cat. no. FG-4592; Selleck Chemicals) were added.

RPTECs cultured under normoxic conditions for a total of $26 \mathrm{~h}$ were used as control. Two hours before the end of the 26-h period, control cells were washed with PBS and fresh culture medium was added.

Cell imaging. Cell images were captured at the onset of reoxygenation, then at 2-h intervals for $24 \mathrm{~h}$. An Axiovert 40C inverted microscope (Carl Zeiss AG) equipped with a digital camera (3MP USB2.0 Microscope Digital Camera; Amscope) and related software (Amscope; v. x64, 3.7.3036) were used.

Assessment of ROS production, lipid peroxidation and cell necrosis. To assess ROS production, RPTECs were cultured in 96-well plates. At the end of the reoxygenation period, $5 \mu \mathrm{M}$ of the fluorogenic probe CellROX ${ }^{\circledR}$ Deep Red Reagent (Invitrogen; Thermo Fisher Scientific, Inc.) was added, and cells were incubated at $37^{\circ} \mathrm{C}$ for $30 \mathrm{~min}$. Then, cells were washed with PBS, and fluorescence signal intensity was measured with an EnSpire ${ }^{\circledR}$ Multimode plate reader (PerkinElmer, Inc.).

To assess lipid peroxidation, RPTECs were cultured in 6-well plates. At the end of the reoxygenation period, the end product of lipid peroxidation malondialdehyde (MDA) was measured fluorometrically in cell lysates using the Lipid Peroxidation (MDA) Assay Kit (cat. no. ab118970; Abcam). The required cell lysis buffer was provided along with the aforementioned kit. The lower detection limit of the kit is $0.1 \mathrm{nmol}$. Prior to MDA measurement, a Bradford assay was performed to adjust lysate volumes to an equal protein mass of $100 \mu \mathrm{g}$.

To assess cell necrosis and the effect of AhR, RPTECs were cultured in 96-well plates in the presence or absence of the ferroptosis inhibitor $\alpha$-tocopherol or the AhR inhibitor $\mathrm{CH} 223191$. The above inhibitors were added into the cell cultures at the beginning of the reoxygenation period. At the end of the reoxygenation period, cell necrosis was evaluated using a lactate dehydrogenase (LDH) release assay with the Cytotox Non-Radioactive Cytotoxic Assay kit (Promega Corporation). Cell necrosis was calculated as (LDH in the supernatant/Total LDH) x 100.

Western blot analysis. RPTECs were cultured in 6-well plates. Once the reoxygenation period was over, cells were lysed with the T-PER tissue protein extraction reagent (Thermo Fisher Scientific, Inc.) supplemented with phosphatase inhibitor (Roche Diagnostics) and protease inhibitor (Sigma-Aldrich; Merck KGaA). Protein was quantified using a Bradford assay (Sigma-Aldrich; Merck KGaA). For western blotting, $10 \mu \mathrm{g}$ of protein from each sample were electrophoresed in SDS-PAGE (4-12\% Bis-Tris) gels (Thermo Fisher Scientific, Inc.) and transferred to PVDF membranes (Thermo Fisher Scientific, Inc.). Tris-buffered saline with $0.1 \%$ Tween- 20 and 5\% non-fat dry milk was used as blocking buffer. Blots were incubated at $4^{\circ} \mathrm{C}$ for $16 \mathrm{~h}$ with the primary antibody, then at room temperature for $30 \mathrm{~min}$ with the secondary antibody. For the enhanced chemiluminescent detection of the western blot bands, the LumiSensor Plus Chemiluminescent HRP Substrate kit (GenScript Corporation) was used. Whenever reprobing of the PVDF blots was necessary, the Restore Western Blot Stripping Buffer (Thermo Fisher Scientific, Inc.) was used. The Image J software v. 1.51t (National Institutes of Health) was used for densitometric analysis.

Primary antibodies were specific for AhR (1:200; cat. no. sc-133088; Santa Cruz Biotechnology, Inc.), cytochrome P450 family 1 subfamily A member 1 (CYP1A1; 1:500; cat. no. sc-25304; Santa Cruz Biotechnology, Inc.), Nrf2 (1:1,000; cat. no. TA343586; OriGene Technologies, Inc.), superoxide dismutase 3 (SOD-3; 1:100; cat. no. sc-271170; Santa Cruz Biotechnology, Inc.), cystine-glutamate antiporter (xCT, also known as SLC7A11; 1:1,000; cat. no. ANT-111; Alomone Labs), HIF-1 $\alpha$ (1:500; cat. no. sc-10790; Santa Cruz Biotechnology, Inc.), LDH-A (1:1,000; cat. no. 2012; Cell Signaling Technology, Inc.), activated cleaved caspase-3 (CC3; 1:1,000; cat. no. ab13847; Abcam) and $\beta$-actin (1:2,500; cat. no. 4967; Cell Signaling Technology, Inc.). Anti-mouse IgG, HRP-linked antibody (1:1,000; cat. no. 7076; Cell Signaling Technology, Inc.) or anti-rabbit IgG, HRP-linked antibody (1:1,000; cat. no. 7074; Cell Signaling Technology, Inc.) were used as secondary antibodies.

Statistical analysis. One-sample Kolmogorov-Smirnov test verified that all, except one, variables were normally distributed. One-way analysis of variance and Bonferroni's correction test were used for comparison of means. Results are presented as the mean \pm SEM of six experiments. The analysis of the cell imaging results was carried out using the Kruskal-Wallis $\mathrm{H}$ test and Dunn's post hoc test, since this variable did not follow the normal distribution. Statistical analysis was performed with the SPPS version 20 (IBM Corp.). $\mathrm{P}<0.05$ was considered to indicate a statistically significant difference.

\section{Results}

Reoxygenation activates AhR and increases CYPIAl expression, while CH223191 prevents both. Reoxygenation decreased AhR levels to $0.68 \pm 0.05$ of the control $(P=0.001)$. The AhR inhibitor $\mathrm{CH} 223191$ inhibited the reoxygenation-induced changes in AhR levels, increasing the AhR levels $0.99 \pm 0.08$ of the control (P not significant compared with the control; $\mathrm{P}=0.003$ compared with cells subjected to reoxygenation only). Of note, under normoxic conditions, $\mathrm{CH} 223191$ increased AhR level to $1.29 \pm 0.04$ of the control $(P=0.03)$. Since AhR is degraded after its activation, these results indicated that reoxygenation activated $\mathrm{AhR}$, and that this effect was inhibited by the AhR specific inhibitor CH223191 (Fig. 1A and B).

The expression of the AhR transcriptional target CYP1A1 confirmed the aforementioned conclusion. Reoxygenation increased CYP1A1 expression to $1.44 \pm 0.01$ of the control $(\mathrm{P}=0.033) . \mathrm{CH} 223191$ prevented the reoxygenation-induced change of CYP1A1 expression since, in this case, CYP1A1 level equaled $1.04 \pm 0.07$ of the control ( $P$ not significant compared with the control; $\mathrm{P}=0.014$ compared with cells subjected to reoxygenation only). Notably, under normoxic conditions, $\mathrm{CH} 223191$ decreased CYP1A1 expression levels to $0.66 \pm 0.05$ of the control $(\mathrm{P}=0.033$; Fig. $1 \mathrm{~A}$ and $\mathrm{C})$. 


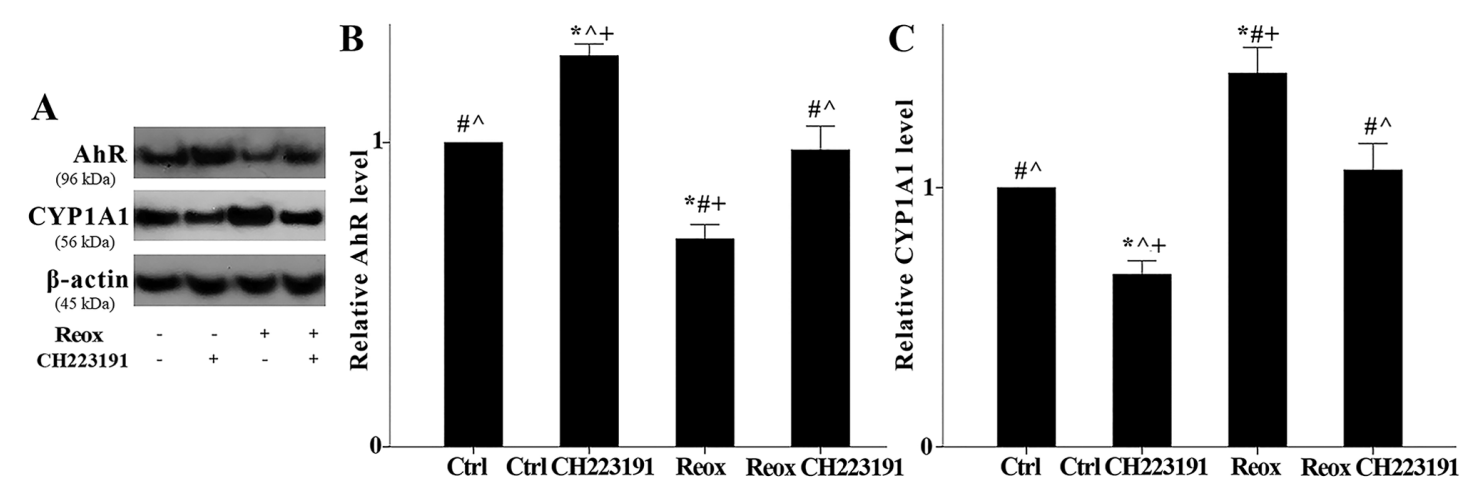

Figure 1. Reox activates AhR and induces CYP1A1 expression, while CH223191 inhibits both. RPTECs were cultured under ctrl conditions or subjected to Reox in the presence or absence of the AhR inhibitor CH223191. (A) Representative AhR and CYP1A1 western blot images. (B and C) Statistical analysis of the western blots. Reox decreases AhR levels, indicating its activation. CH223191 increases AhR levels both in ctrl cells and cells subjected to Reox. Reox enhanced CYP1A1 expression, whereas CH223191 reduced CYP1A1 under both conditions (C). Data are presented as the mean \pm SEM of six independent experiments. " $\mathrm{P}<0.05$ vs. ctrl; ${ }^{*} \mathrm{P}<0.05$ vs. ctrl $\mathrm{CH} 223191$; ${ }^{\circ} \mathrm{P}<0.05$ vs. Reox; ${ }^{+} \mathrm{P}<0.05$ vs. Reox $\mathrm{CH} 223191$. AhR, arylhydrocarbon receptor; CYP1A1, cytochrome P450 family 1 subfamily A member 1; RPTEC, renal proximal tubular epithelial cell; ctrl, control; Reox, reoxygenation.
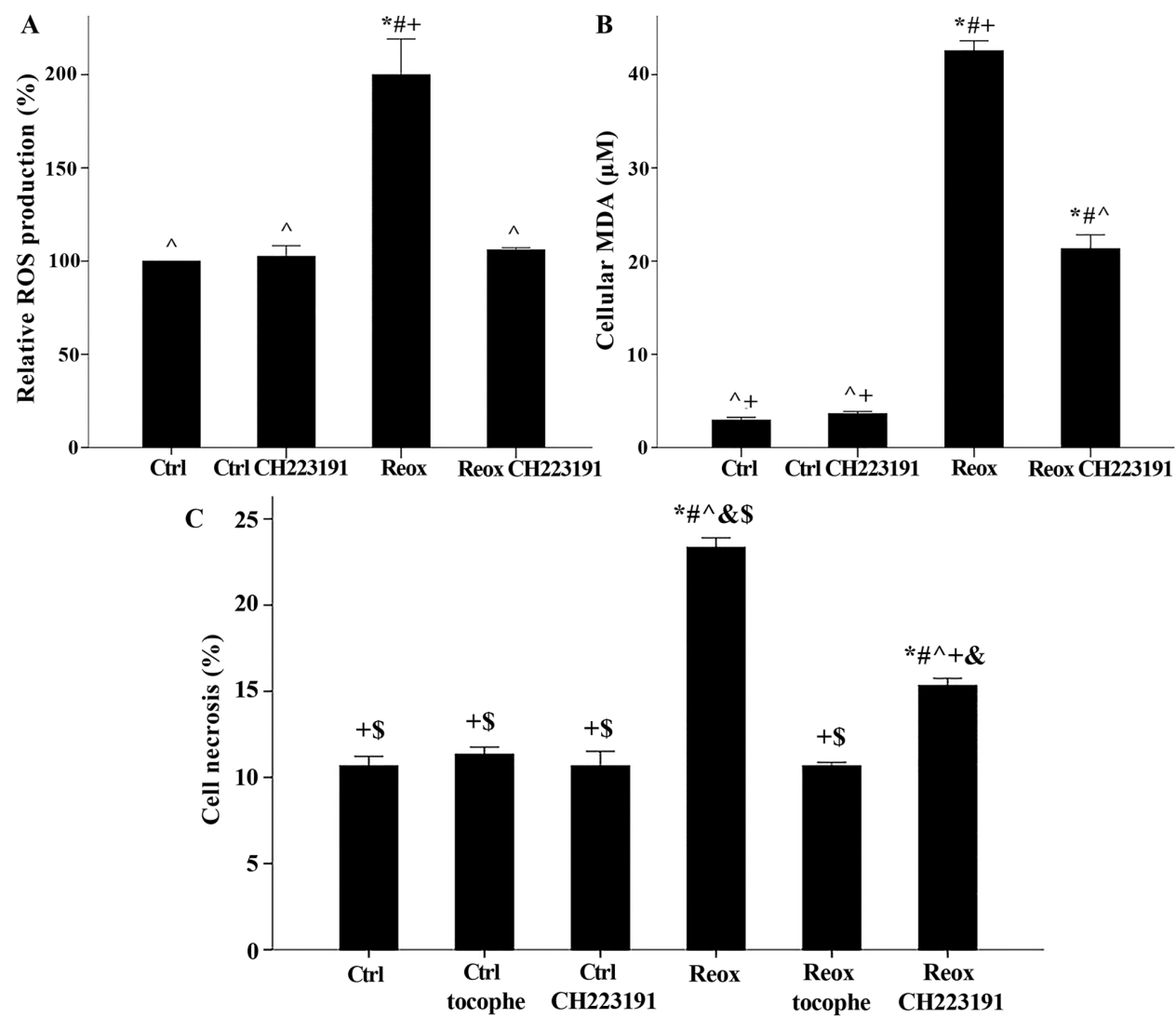

Figure 2. Inhibition of AhR prevents Reox-induced ROS production and attenuates lipid peroxidation and ferroptosis. RPTECs were cultured under ctrl conditions or subjected to Reox, with or without the AhR inhibitor CH223191 or the ferroptosis inhibitor tocophe. (A and B) Reox induced ROS production and lipid peroxidation, assessed by MDA levels. CH223191 prevented Reox-induced ROS production and attenuated lipid peroxidation. Reox caused cell necrosis. Necrosis was abrogated by $\alpha$-tocopherol, demonstrating ferroptosis. ${ }^{*} \mathrm{P}<0.05$ vs. ctrl; ${ }^{*} \mathrm{P}<0.05$ vs. ctrl $\mathrm{CH} 223191 ;{ }^{\wedge} \mathrm{P}<0.05$ vs. Reox; ${ }^{+} \mathrm{P}<0.05$ vs. Reox $\mathrm{CH} 223191$. (C) CH223191 ameliorated Reox-induced cell necrosis. Data are presented as the mean \pm SEM of six independent experiments. ${ }^{*} \mathrm{P}<0.05 \mathrm{vs}$. ctrl; ${ }^{\#} \mathrm{P}<0.05$ vs. ctrl tocophe; ${ }^{\wedge} \mathrm{P}<0.05$ vs. $\operatorname{ctrl} \mathrm{CH} 223191 ;{ }^{+} \mathrm{P}<0.05$ vs. Reox, ${ }^{\circledR} \mathrm{P}<0.05$ vs. Reox tocophe; ${ }^{\$} \mathrm{P}<0.05$ vs. Reox $\mathrm{CH} 223191$. AhR, arylhydrocarbon receptor; RPTEC, renal proximal tubular epithelial cell; ROS, reactive oxygen species; MDA, malondialdehyde; ctrl, control; Reox, reoxygenation; tocophe; $\alpha$-tocopherol.

Inhibition of AhR prevents reoxygenation-induced ROS production and ameliorates lipid peroxidation. Reoxygenation increased ROS production. Considering the ROS production in control cells as $100 \%$, in cells subjected to reoxygenation, ROS levels doubled, compared with the control $(199.9 \pm 9.6 \%$; $\mathrm{P}<0.001)$. The AhR inhibitor $\mathrm{CH} 223191$ prevented reoxygen- ation-induced ROS production, with ROS level only reaching $106.0 \pm 0.6 \%$ of the control (P not significant compared with the control; $\mathrm{P}<0.001$ compared with cells subjected to reoxygenation only). Under normoxic conditions, $\mathrm{CH} 223191$ did not affect ROS production $(102.5 \pm 2.9 \%$ of the control; P not significant; Fig. 2A). 


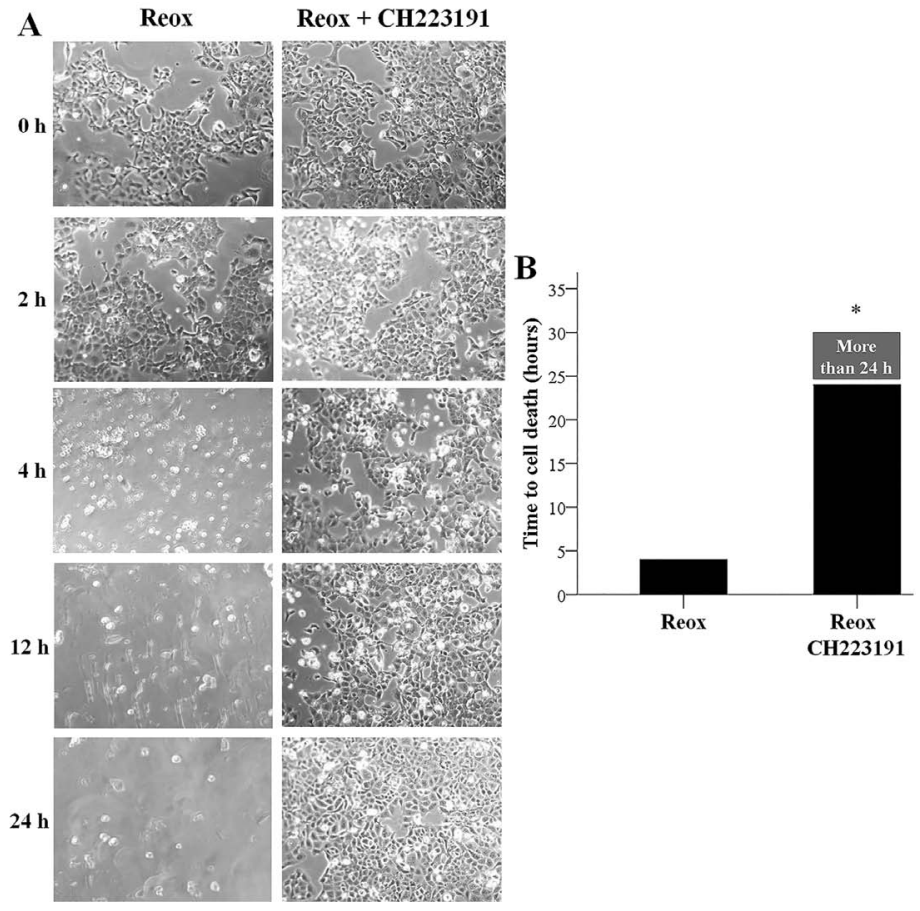

Figure 3. Inhibition of AhR protects from Reox-induced cell death. RPTECs were subjected to Reox with or without the AhR inhibitor CH223191. (A) Representative images of one of the experiments. Magnification, x100. (B) Statistical analysis of all experiments. RPTECs died within $4 \mathrm{~h}$ of Reox, while CH223191 rescued the cells. Data are presented as the mean \pm SEM of six independent experiments. " $\mathrm{P}<0.05$ vs. Reox. AhR, arylhydrocarbon receptor; RPTEC, renal proximal tubular epithelial cell; Reox, reoxygenation.

A

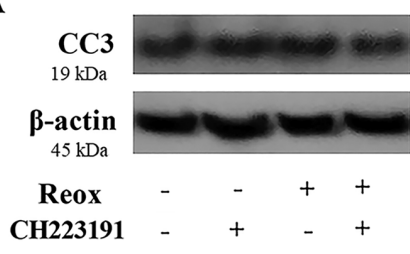

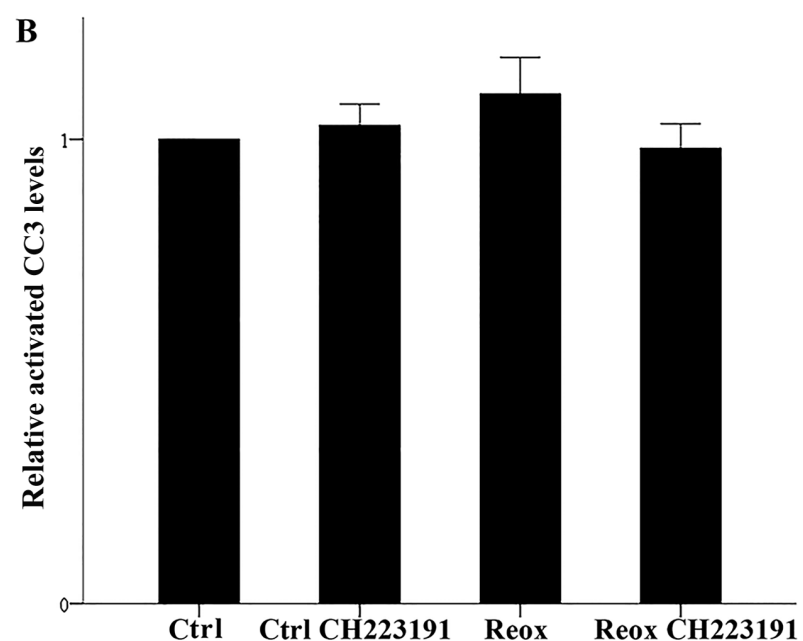

Figure 4. Apoptosis does not play a role in Reox-induced cell death and is not affected by inhibition of AhR. RPTECs were cultured under ctrl conditions or subjected to Reox in the presence or not of the AhR inhibitor CH223191. (A) Representative CC3 western blot images. (B) Statistical analysis of the western blots. Neither Reox nor CH223191 affects cell apoptosis. Data are presented as the mean \pm SEM of six independent experiments. AhR, arylhydrocarbon receptor; RPTEC, renal proximal tubular epithelial cell; ctrl, control; Reox, reoxygenation; CC3, cleaved caspase-3.

Lipid peroxidation, assessed by MDA levels, followed the same trend as ROS. In control cells, cellular MDA concentration was $3.0 \pm 0.2 \mu \mathrm{M}$, while under reoxygenation, MDA concentration increased to $42.6 \pm 0.5 \mu \mathrm{M}(\mathrm{P}<0.001)$. $\mathrm{CH} 223191$ decreased MDA in RPTECs subjected to reoxygenation to $21.3 \pm 0.7 \mu \mathrm{M}$ $(\mathrm{P}<0.001$, compared with cells subjected to reoxygenation only). Notably, inhibition of AhR under control conditions did not alter MDA significantly (3.6 $60.3 \mu \mathrm{M}$; Fig. 2B).

Inhibition of AhR attenuates reoxygenation-induced ferroptosis, while apoptosis was not observed. Cell imaging indicated that RPTECs were extremely vulnerable to reoxygenation since in all performed experiments they died after $4 \mathrm{~h}$. The specific AhR inhibitor CH223191 rescued RPTECs from reoxygenation induced cell death since, in this case, in all experiments, cells remained alive at the 24 -h timepoint $(\mathrm{P}<0.001$; Fig. $3 \mathrm{~A}$ and $\mathrm{B})$.

In the control cells, LDH release assay indicated a percentage of necrotic cells equal to $10.7 \pm 0.6 \%$. In cells subjected to reoxygenation, the percentage of necrotic cells increased significantly to $23.3 \pm 0.6 \%$, compared with control cells $(\mathrm{P}<0.001)$. The inhibitor of ferroptosis $\alpha$-tocopherol 

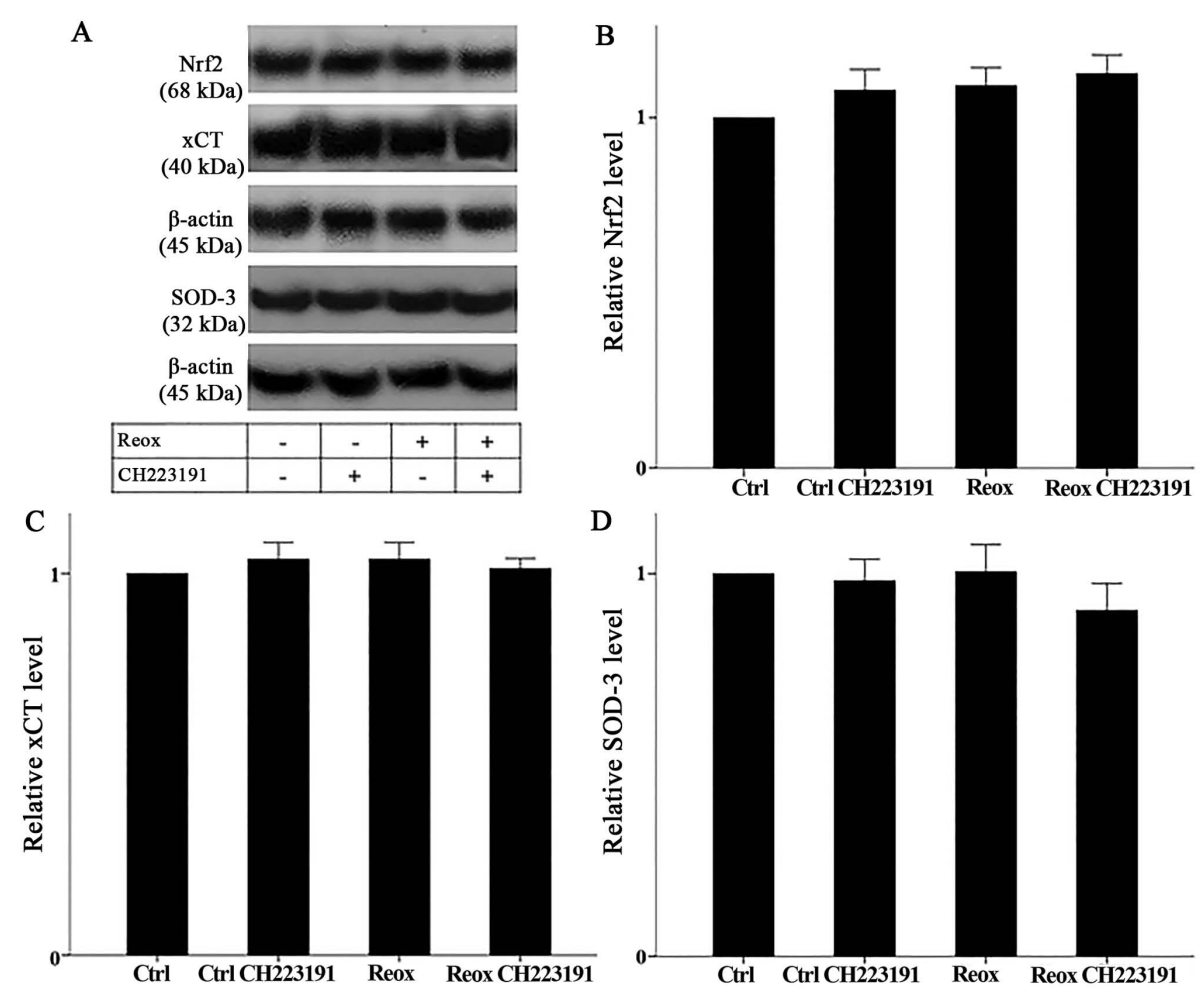

Figure 5. AhR activation status does not affect Nrf2 activation or transcriptional activity. RPTECs were cultured under ctrl conditions or subjected to Reox with or without the AhR inhibitor CH223191. (A) Representative western blots of Nrf2 levels (corresponding to its activation status) and the expression of the Nrf2 transcriptional targets xCT (SLC7A11) and SOD-3. (B-D) Statistical analysis of the western blots. Neither Reox nor CH223191 affects Nrf2 activity, or the expression of $\mathrm{xCT}$ and SOD-3. Data are presented as the mean \pm SEM of six independent experiments. AhR, arylhydrocarbon receptor; Nrf2, nuclear factor erythroid 2-related factor 2; xCT, cystine-glutamate antiporter; SOD-3, superoxide dismutase; RPTEC, renal proximal tubular epithelial cell; ctrl, control; Reox, reoxygenation.
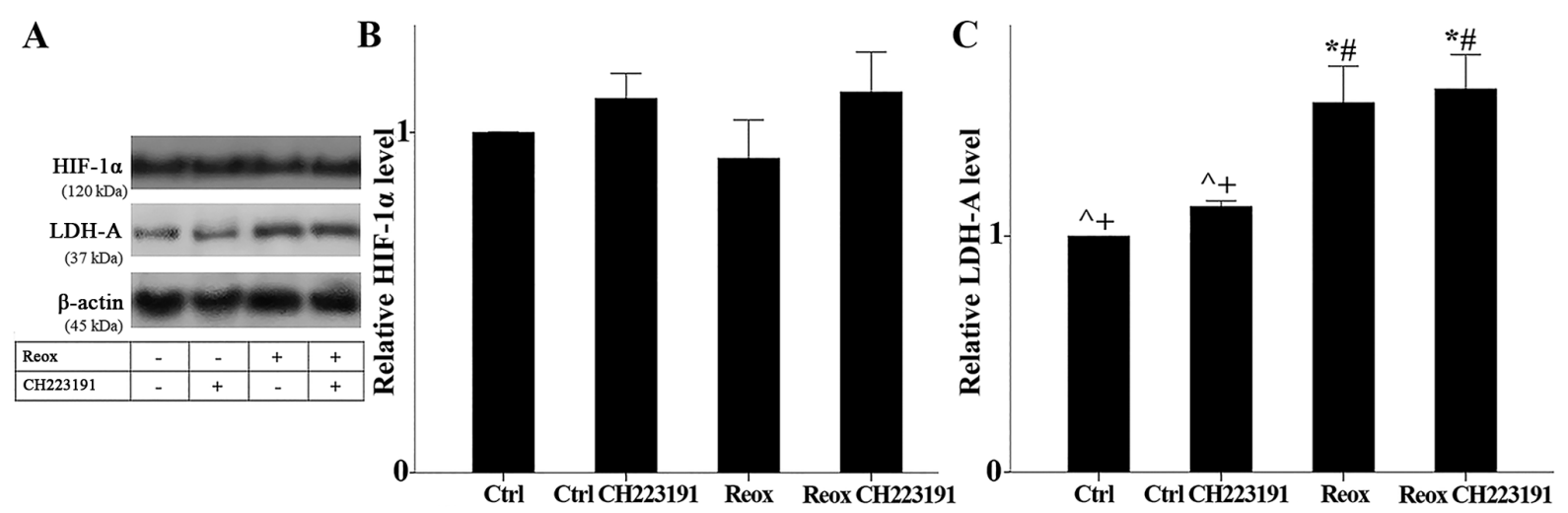

Figure 6. AhR activation status does not affect HIF-1 $\alpha$ levels or its transcriptional activity. RPTECs were cultured under ctrl conditions or subjected to Reox with or without the AhR inhibitor CH223191. (A) Representative western blot images of HIF-1 $\alpha$ levels (corresponding to its activation status) and its transcriptional target LDH-A. (B and C) Statistical analysis of the western blots. Neither Reox nor CH223191 affected HIF-1 $\alpha$ levels. LDH-A levels increased under Reox, yet $\mathrm{CH} 223191$ did not affect LDH-A expression in both ctrl cells and cells subjected to Reox. Data are presented as the mean \pm SEM. ${ }^{*} \mathrm{P}<0.05$ vs. ctrl; ${ }^{\mathrm{P}} \mathrm{P}<0.05$ vs. ctrl CH223191; ${ }^{\wedge} \mathrm{P}<0.05$ vs. Reox; ${ }^{+} \mathrm{P}<0.05$ vs. Reox $\mathrm{CH} 223191$. AhR, arylhydrocarbon receptor; RPTEC, renal proximal tubular epithelial cell; HIF-1 $\alpha$, hypoxia-inducible factor $1 \alpha$; LDH-A, lactate dehydrogenase-A; ctrl, control; Reox, reoxygenation.

prevented reoxygenation-induced cell necrosis completely since, in this case, the percentage of cell necrosis was equal to that detected in control cells $(10.7 \pm 0.2 \%$; P not significant compared to the control cells; $\mathrm{P}<0.001$ compared to cells subjected to reoxygenation only). Thus, reoxygenation-induced cell necrosis may be mediated through the ferroptotic pathway. Moreover, the AhR inhibitor CH223191 ameliorated reoxygenation-induced cell necrosis considerably. Compared with the cells subjected to reoxygenation, in cells subjected to reoxygenation and treated with the AhR inhibitor, cell necrosis percentage decreased from $23.3 \pm 0.6$ to $15.3 \pm 0.4 \%(\mathrm{P}<0.001)$. Of note, under control conditions, $\mathrm{CH} 223191$ was not toxic for RPTECs, since the percentage of cell necrosis was $10.7 \pm 0.8 \%$ (P not significant; Fig. 2C).

Apoptosis was assessed using the levels of CC3. Neither reoxygenation nor $\mathrm{CH} 223191$ had any effect on $\mathrm{CC} 3$ levels. Considering the level of CC3 in the control cells as 1, CC3 level equaled $1.0 \pm 0.05$ in cells treated with the inhibitor, 

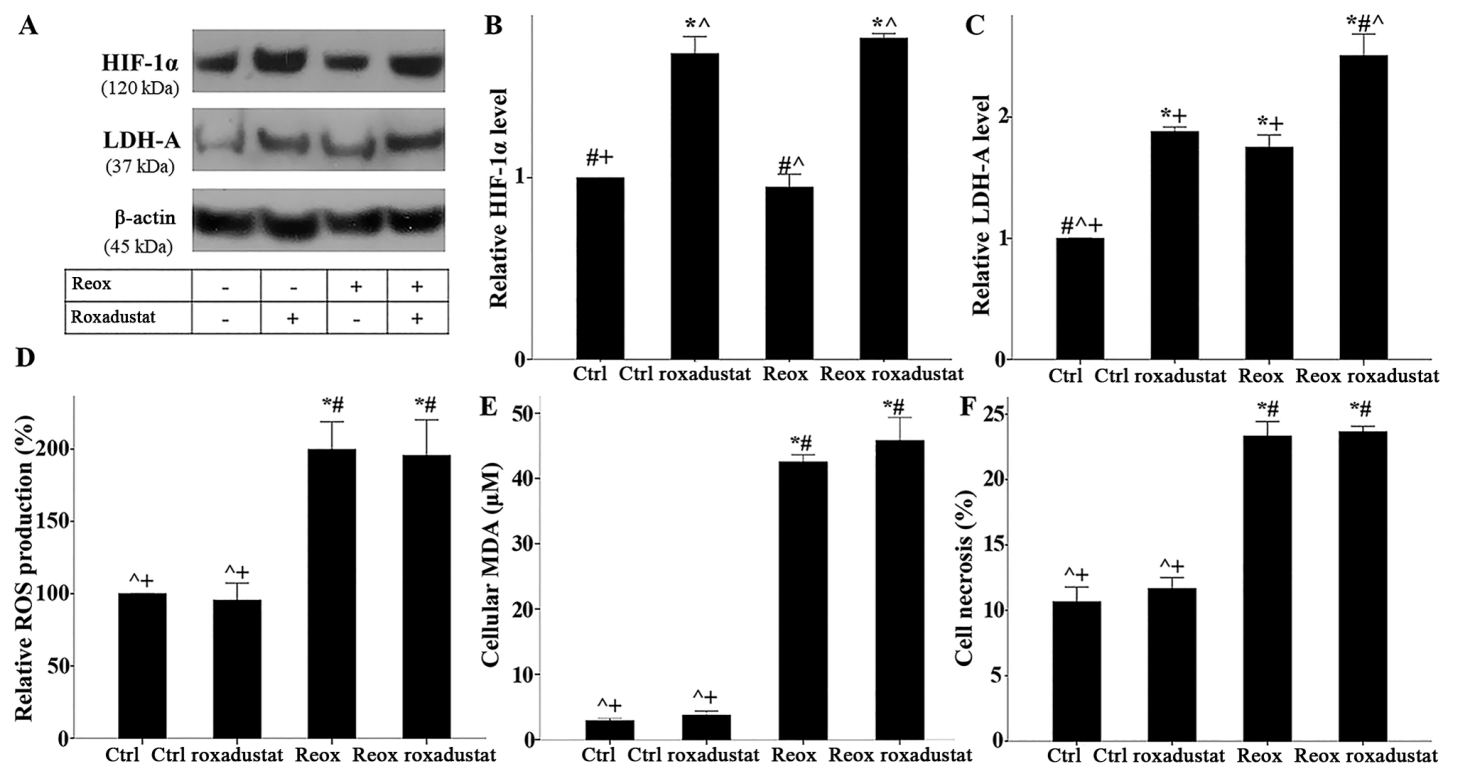

Figure 7. Roxadustat increases HIF-1 $\alpha$ level and transcriptional activity but does not affect ROS production, lipid peroxidation, or cell necrosis. RPTECs were cultured under ctrl conditions or subjected to Reox with or without the HIF-1 $\alpha$ activator roxadustat. (A) Representative western blot images of HIF-1 $\alpha$ levels (corresponding to its activation status) and its transcriptional target LDH-A. (B and C) Statistical analysis of the western blots. Roxadustat enhanced HIF-1 $\alpha$ levels in both ctrl cells and cells subjected to Reox. Roxadustat also upregulated LDH-A expression in both ctrl cells and cells subjected to Reox. Compared to ctrl cells, LDH-A levels increased in cells subjected to Reox. (D) ROS production, (E) lipid peroxidation and (F) cell necrosis were also assessed. Roxadustat did not affect Reox-induced ROS production, lipid peroxidation, and cell necrosis. Data are presented as the mean $\pm \mathrm{SEM}$. $\mathrm{P}<0.05$ vs. ctrl; ${ }^{\prime} \mathrm{P}<0.05 \mathrm{vs}$. ctrl roxadustat; ${ }^{\wedge} \mathrm{P}<0.05$ vs. Reox; ${ }^{+} \mathrm{P}<0.05$ vs. Reox roxadustat. RPTEC, renal proximal tubular epithelial cell; HIF-1 $\alpha$, hypoxia-inducible factor $1 \alpha ; \mathrm{LDH}-\mathrm{A}$, lactate dehydrogenase-A; ROS, reactive oxygen species; MDA, malondialdehyde; ctrl, control; Reox, reoxygenation.

$1.1 \pm 0.08$ in RPTECs subjected to reoxygenation, and $1.0 \pm 0.03$ in cells subjected to reoxygenation and treated with $\mathrm{CH} 223191$ (P not significant; Fig. 4).

AhR activation status does not affect Nrf2 activation or transcriptional activity. Neither reoxygenation nor the AhR inhibitor CH223191 affected Nrf2 levels (which correspond to its activation). In RPTECs subjected to reoxygenation, Nrf2 levels equaled $1.01 \pm 0.07$ of the control ( $\mathrm{P}$ not significant). In RPTECs subjected to reoxygenation and treated with $\mathrm{CH} 223191, \mathrm{Nrf} 2$ levels equaled $0.97 \pm 0.03$ of the control (P not significant, compared with control cells). Under normoxia, RPTECs treated with $\mathrm{CH} 223191, \mathrm{Nrf} 2$ levels equaled $0.98 \pm 0.06$ of the control (P not significant; Fig. 5A and B). Thus, AhR does not affect Nrf2 activation status.

The stable expression of the Nrf2 transcriptional targets $\mathrm{xCT}$ and SOD-3 confirmed the aforementioned conclusion. Under reoxygenation, $\mathrm{xCT}$ and SOD-3 expression remained stable and equaled $1.04 \pm 0.04$ and $1.01 \pm 0.07$ of the control, respectively ( $\mathrm{P}$ not significant in both cases). In RPTECs subjected to reoxygenation and treated with $\mathrm{CH} 223191$, $\mathrm{xCT}$ and SOD-3 expression also remained stable. In this case, $\mathrm{xCT}$ and SOD-3 levels equaled $1.01 \pm 0.03$ and $0.91 \pm 0.07$ of the control, respectively ( $\mathrm{P}$ not significant in both cases). Moreover, under normoxic conditions, $\mathrm{CH} 223191$ did not alter the expression of the above proteins. $\mathrm{xCT}$ and SOD-3 expression levels equaled $1.04 \pm 0.04$ and $0.98 \pm 0.06$ of the control, respectively ( $\mathrm{P}$ not significant in both cases; Fig. 5A, C and D).

AhR activation status does not affect HIF-1a levels or its transcriptional targets. Neither reoxygenation nor $\mathrm{CH} 223191$ affected HIF-1 $\alpha$ levels (which correspond to its activation). In cells treated with the AhR inhibitor $\mathrm{CH} 223191$, cells subjected to reoxygenation, and cells subjected to reoxygenation and treated with $\mathrm{CH} 223191$, HIF-1 $\alpha$ levels were $1.10 \pm 0.07$, $0.92 \pm 0.11$, and $1.12 \pm 0.12$ of the control, respectively ( $\mathrm{P}$ not significant in any case; Fig. 6A and B). Thus, AhR does not alter HIF-1 $\alpha$ activity.

The AhR inhibitor $\mathrm{CH} 223191$ did not affect the expression of the HIF-1 $\alpha$ transcriptional target LDH-A, confirming the aforementioned conclusion. Compared to the control cells, in cells treated with $\mathrm{CH} 223191$, LDH-A expression remained stable $(1.12 \pm 0.02$ of the control; P not significant). Reoxygenation increased LDH-A expression to $1.56 \pm 0.16$ of the control $(\mathrm{P}=0.008)$. However, $\mathrm{CH} 223191 \mathrm{did}$ not result in any further change to LDH-A expression, $(1.62 \pm 0.15$ of the control; P not significant compared with reoxygenation only; Fig. 6A and C).

Roxadustat increases HIF-1 $\alpha$ level and transcriptional activity but does not affect ROS production, lipid peroxidation, or cell survival. Under normoxia, roxadustat increased HIF-1 $\alpha$ to $1.68 \pm 0.09$ of the control $(\mathrm{P}<0.001)$. Reoxygenation did not alter HIF-1 $\alpha$, compared with the control cells $(0.95 \pm 0.07$ of the control; P not significant). In RPTECs subjected to reoxygenation and treated with roxadustat, HIF-1 $\alpha$ increased to $1.77 \pm 0.03$ of the control $(\mathrm{P}<0.001$, compared with the control and cells subjected to reoxygenation alone; Fig. 7A and B). Thus, the HIF-1 $\alpha$ activator roxadustat increased HIF-1 $\alpha$ level both in control cells and in cells subjected to reoxygenation.

Similarly, roxadustat increased the expression of the HIF-1 $\alpha$ transcriptional target LDH-A, confirming that this compound activates HIF- $1 \alpha$ at the used concentration of $10 \mu \mathrm{g} / \mathrm{ml}$. Under normoxic conditions, roxadustat enhanced LDH-A expression 

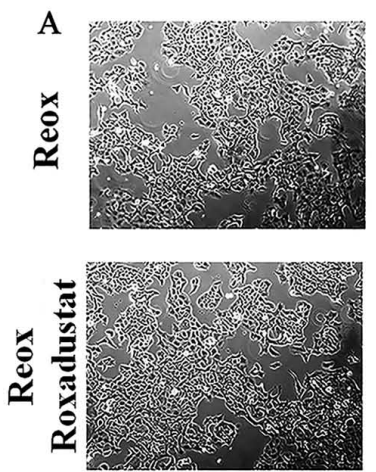

$0 \mathrm{~h}$
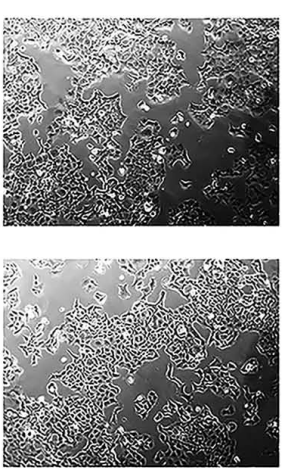

$2 \mathrm{~h}$
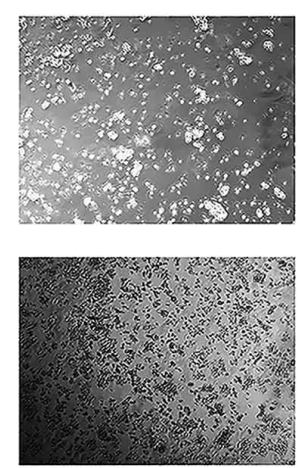

$4 \mathrm{~h}$

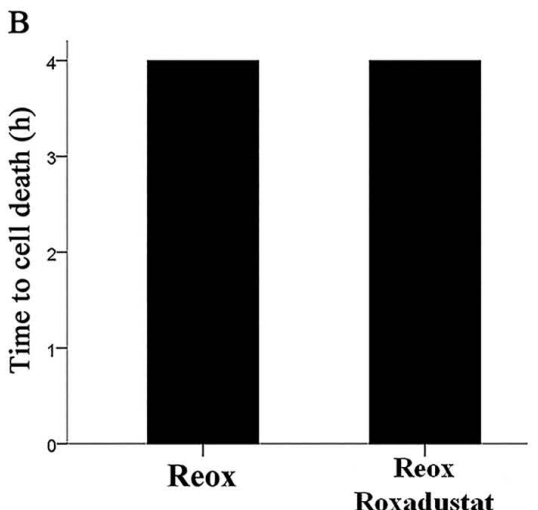

Figure 8. Activation of HIF-1 $\alpha$ protects from Reox-induced cell death. RPTECs were subjected to Reox with or without the HIF-1 $\alpha$ activator roxadustat. (A) Representative images of one of the experiments. Magnification, x100. (B) Statistical analysis of all experiments. Roxadustat did not alter RPTEC resistance to Reox-induced cell death, since both roxadustat-treated and untreated cells died within $4 \mathrm{~h}$ of observation. Data are presented as the mean \pm SEM of six independent experiments. RPTEC, renal proximal tubular epithelial cell; HIF-1 $\alpha$, hypoxia-inducible factor $1 \alpha$; Reox, reoxygenation.

to $1.88 \pm 0.04$ of the control $(\mathrm{P}<0.001$, compared with control cells). Reoxygenation increased LDH-A to $1.75 \pm 0.10$ of the control $(\mathrm{P}<0.001$, compared with control cells), although roxadustat treatment of RPTECs subjected to reoxygenation further increased LDH-A expression to $2.51 \pm 0.18$ of the control $(\mathrm{P}<0.001$, compared to control cells and cells subjected to reoxygenation alone; Fig. 7A and $\mathrm{C}$ ).

Roxadustat did not affect ROS production or lipid peroxidation. Treatment of control cells with roxadustat did not alter relative ROS signal intensity. Considering the ROS production in the control cells as $100 \%$, ROS levels reached $95.6 \pm 5.9 \%$ of the control in cells under normoxia and treated with roxadustat (P not significant). Reoxygenation increased ROS signal intensity to $199.9 \pm 9.6 \%$ ( $\mathrm{P}<0.001$, compared with control cells), a value that remained stable when roxadustat was administered (195.7 $\pm 12.3 \%$, P not significant, compared with cells subjected to reoxygenation alone; Fig. 7D). In RPTECs treated with roxadustat, cellular MDA did not change significantly, compared with the control group (3.0 $\pm 0.2 \mu \mathrm{M}$ and 3.6 \pm 0.6 , respectively; $\mathrm{P}$ not significant). Reoxygenation increased MDA concentration to $42.6 \pm 0.5 \mu \mathrm{M}$ ( $\mathrm{P}<0.001$, compared to the control cells). When roxadustat was administered at the onset of reoxygenation, MDA did not alter considerably $(45.8 \pm 1.8 \mu \mathrm{M}, \mathrm{P}$ not significant., compared to cells subjected to reoxygenation only; Fig. 7E).

As regards the hard end-point of cell survival, LDH release assay revealed that in control cells, roxadustat did not alter the percentage of cell necrosis compared with the control $(10.7 \pm 0.6$ vs. $11.7 \pm 0.4 \%$, respectively; P not significant). Reoxygenation increased cell necrosis to $23.3 \pm 0.6 \%$ ( $\mathrm{P}<0.001$, compared to the control cells), while roxadustat did not affect necrosis $(23.7 \pm 0.2 \%$; $\mathrm{P}$ not significant compared with cells subjected to reoxygenation only; Fig. 7F). Cell imaging confirmed the above LDH release assay results since it showed that roxadustat treatment did not offer any survival benefit in RPTECs subjected to reoxygenation. In all performed experiments, both treated and untreated cells died within $4 \mathrm{~h}$ of reoxygenation (P not significant; Fig. 8).

\section{Discussion}

I-R injury contributes to the pathogenesis of many diseases, including the acute kidney injury (1-4). As a consequence, clarifying the pathogenesis of I-R injury is decisive in developing novel therapeutic strategies. This study aimed to evaluate the role of AhR during the reoxygenation phase of I-R injury.

Some data support a detrimental role of AhR during the anoxic phase of I-R injury. Inhibition of AhR/CYP1A1 protects murine hippocampal cells from anoxia-induced apoptosis (44). However, no data are available concerning the role of AhR during the subsequent reoxygenation. To evaluate the role of AhR in RPTECs subjected to reoxygenation, a cell culture system designed to study anoxia and reoxygenation separately was established.

In the present study, reoxygenation decreased AhR levels. The decrease in AhR level indicates its activation since activated AhR becomes vulnerable to proteasomal degradation, leading to decreased AhR cellular levels (20-24). Accordingly, reoxygenation increased the expression of CYP1A1, an AhR transcriptional target $(18,19)$. The specific AhR inhibitor CH223191 increased AhR level and decreased CYP1A1 expression. Thus, AhR was activated during reoxygenation, and CH223191 successfully inhibited AhR activation. The exact endogenous AhR ligands, which results in its activation during reoxygenation, remain to be defined. Initially, AhR was considered a critical factor for the CYP-mediated metabolism of xenobiotics, such as dioxins, which are also AhR activators. However, endogenous AhR activators were identified subsequently $(45,46)$. Examples of exogenous AhR activators include natural plant flavonoids, polyphenolics, and indoles, as well as dioxins-like and other synthetic compounds, collectively characterized as xenobiotics. Examples of endogenous AhR ligands include tryptophan derivatives, steroids, eicosanoids, and heme metabolites (45).

Observation of cell cultures under reoxygenation suggested that mouse RPTECs were particularly vulnerable to reoxygenation injury since they died within 4 hours. However, when the AhR inhibitor CH223191 was added at the onset of reoxygenation, it prevented cell death, with the cells remaining alive after $24 \mathrm{~h}$ of culture.

In accordance with previous experiments with the same cell type and similar experimental conditions (5), reoxygenation-induced cell death was not apoptotic since the levels of the activated $\mathrm{CC} 3$, in which all the apoptotic pathways 
converge (47), was not altered. Of note, $\mathrm{CH} 223191 \mathrm{did}$ not enhance CC3, indicating that at the used concentration, this inhibitor does not induce apoptosis in RPTECs.

The primary factor that induces cell injury during the reperfusion phase of I-R is the burst of ROS production, which follows the restoration of oxygen supply to the ischemic tissues (1-3). CYPs play a significant role in ROS production during I-R injury. In experimental models of heart and liver I-R injury, inhibition of CYPs ameliorates ROS production and organ dysfunction (13-17). AhR transcribes certain CYP genes $(18,19)$, and consequently, AhR activation during reoxygenation may contribute to cell injury. Indeed, in experimental models of heart and lung I-R injury, inhibition of AhR was beneficial $(25,35)$. However, in the aforementioned studies, AhR and CYPs were investigated separately, and not as a part of a pathway. Importantly, the aforementioned studies did not discriminate between events taking place under the anoxic phase and from those occurring during the reoxygenation phase of I-R injury. The effect of AhR on ROS production was evaluated in RPTECs subjected to reoxygenation. Reoxygenation induced ROS production, which was prevented by the AhR inhibitor CH223191. Thus, AhR may play a pivotal role in the burst of ROS production that follows reoxygenation.

With regards reoxygenation-induced cell death, previous studies using primary RPTECs and similar experimental conditions have demonstrated that reoxygenation leads to lipid peroxidation and ferroptotic cell death $(5,6)$. Elegant research using isolated murine renal tubules detected the same (7). Interestingly, besides ROS production, CYPs can directly induce lipid peroxidation (48), which causes ferroptosis (43). In our model, reoxygenation increased lipid peroxidation considerably, as identified by cellular MDA. Nevertheless, when AhR was inhibited, reoxygenation-induced lipid peroxidation decreased significantly. In accordance with microscopic observation of cell cultures, the LDH release assay confirmed the reoxygenation-induced cell necrosis biochemically. Administration of the ferroptosis inhibitor $\alpha$-tocopherol prevented reoxygenation-induced cell necrosis completely, indicating the ferroptotic nature of cell death. The inhibition of AhR attenuated reoxygenation-induced cell death considerably, suggesting great therapeutic potential. Of note, in control RPTECs, CH223191 did not induce cell necrosis, proving non-toxic for RPTECs at the used concentration. Thus, during reoxygenation, AhR is activated and increases certain CYPs expression, which in turn, by increasing ROS, induces ferroptotic cell death. The endogenous AhR activators that participate in reoxygenation-induced cell injury remain to be identified. Interestingly, indoleamine 2,3-dioxygenase metabolizes tryptophan to kynurenine, an endogenous AhR activator. Inhibition of this enzyme was protective for murine kidneys subjected to I-R injury. However, whether the beneficial effect of indoleamine 2,3-dioxygenase inhibition was mediated by the suppression of AhR-CYPs pathway was not evaluated (49). The exact impact of indoleamine 2,3-dioxygenase on this pathway deserves further investigation.

Several studies have suggested that AhR induces the expression of Nrf2 (30-32), a transcription factor implicated in the expression of many antioxidant and anti-ferroptotic proteins $(33,34)$. From a teleological point of view, this achieves a balance between AhR-induced CYP-mediated oxidative stress and AhR-induced Nrf2-mediated antioxidant defense $(31,32)$. However, in our model, and despite the reoxygenation-induced AhR activation, the cellular Nrf2 level remained stable. Since activated Nrf2 is released from the ubiquitin ligase cullin-3 and avoids proteasomal degradation, its cellular level corresponds to its activity $(33,34)$. The nuclear Nrf2 levels were not assessed in order to better determine its activation status; instead, the expression of the Nrf2 transcriptional targets SOD-3 and $\mathrm{xCT}$ were measured (33). SOD-3 catalyzes the dismutation of superoxide radical into either molecular oxygen or hydrogen peroxide (50), while xCT enters the necessary for glutathione synthesis cystine into the cell and prevents ferroptosis (43). In the present study, the expression of both Nrf2 transcriptional targets SOD-3 and $\mathrm{xCT}$ remained stable, also indicating that reoxygenation left Nrf2 activation status unaffected. This was in agreement with a previous study showing that reoxygenation failed to enhance Nrf2 activity in mouse RPTECs (51). Interestingly, in RPTECs from the hibernator Syrian hamster, reoxygenation activates Nrf2 contributing to the resistance of these cells to reoxygenation-induced ferroptosis (51). Moreover, in the present study, the specific AhR inhibitor $\mathrm{CH} 223191$ was used. Cell treatment with the AhR inhibitor did not alter Nrf2, SOD-3, and xCT level, offering additional support to the idea that in the context of reoxygenation, AhR does not affect the Nrf2 pathway.

Another transcription factor implicated in I-R is HIF- $1 \alpha$. HIF- $1 \alpha$ is upregulated during the ischemic phase, forms a complex with HIF-1 $\beta$, and transcribes many genes required for adaptation to anoxic conditions. For instance, LDH upregulation promotes the anaerobic catabolism of glucose $(36,37)$. However, activated AhR also associates with HIF-1 $\beta(33,34)$. In certain experimental models, this competition between AhR and HIF- $\alpha$ for HIF- $1 \beta$ results in reduced HIF- $1 \alpha$ transcriptional activity in the case of AhR activation, or to decreased AhR transcriptional activity in the case of HIF-1 $\alpha$ activation (38-40). However, the interaction between AhR and HIF-1 has not been evaluated during the reperfusion phase of I-R injury. In the present experimental model, inhibition of AhR during reoxygenation did not affect either HIF-1 $\alpha$ levels $(36,37)$, or the expression of its transcriptional target LDH-A. Interestingly, in RPTECs under reoxygenation, HIF-1 $\alpha$ level was similar to the control cells, whereas the expression of LDH-A was increased. The latter may result from rapid HIF-1 $\alpha$ degradation when oxygen is available. By contrast, the increased LDH-A levels may be the result of a slower turnover of this enzyme, which is upregulated during the anoxic phase.

To delineate the role of HIF-1 $\alpha$ further, RPTECs were subjected to reoxygenation with the HIF- $1 \alpha$ activator roxadustat. As expected, roxadustat increased HIF- $1 \alpha$ levels and LDH-A expression in both control cells and cells subjected to reoxygenation. However, roxadustat did not affect ROS production, lipid peroxidation or cell death. Thus, during reoxygenation, HIF-1 $\alpha$ does not play a pivotal role in cell survival.

Certainly, the in vitro nature of our study is a limitation. However, this approach allowed us to apply strict experimental conditions and to evaluate the effect of AhR on cell survival solely during the reoxygenation phase. As already noted, I-R injury consists of two consecutive but pathophysiologically distinct phases, anoxia and reoxygenation (1-3). A better understanding of the molecular mechanisms that govern the 
two stages of I-R injury may result in an efficient combination of therapeutic approaches targeting both stages.

In conclusion, in RPTECs, AhR is activated during reoxygenation and induces ROS production, lipid peroxidation and ferroptotic cell death. These detrimental effects may be mediated by AhR-induced CYP overexpression, while AhR does not affect the Nrf2 or the HIF-1 $\alpha$ pathways. Thus, the AhR pathway may represent a promising therapeutic target for the prevention of reperfusion injury.

\section{Acknowledgements}

Not applicable.

\section{Funding}

No funding was received.

\section{Availability of data and materials}

The datasets used and/or analyzed during the current study are available from the corresponding author on reasonable request.

\section{Authors' contributions}

TE designed the study. GP and TE performed the experiments. TE, GP, GF, VL and IS analyzed the results. TE wrote the manuscript with help from GP. All authors read and approved the final manuscript.

\section{Ethics approval and consent to participate}

Not applicable.

\section{Patient consent for publication}

Not applicable.

\section{Competing interests}

The authors declare that they have no competing interests.

\section{References}

1. Neri M, Riezzo I, Pascale N, Pomara C and Turillazzi E: Ischemia/reperfusion injury following acute myocardial infarction: a critical issue for clinicians and forensic pathologists. Mediators Inflamm 2017: 7018393, 2017.

2. Bakthavachalam P and Shanmugam PST: Mitochondrial dysfunction - silent killer in cerebral ischemia. J Neurol Sci 375: 417-423, 2017.

3. Tsukamoto T, Chanthaphavong RS and Pape HC: Current theories on the pathophysiology of multiple organ failure after trauma. Injury 41: 21-26, 2010.

4. Bonventre JV and Yang L: Cellular pathophysiology of ischemic acute kidney injury. J Clin Invest 121: 4210-4221, 2011.

5. Eleftheriadis T, Pissas G, Antoniadi G, Liakopoulos V and Stefanidis I: Cell death patterns due to warm ischemia or reperfusion in renal tubular epithelial cells originating from human, mouse, or the native hibernator hamster. Biology (Basel) 7: 48, 2018.

6. Eleftheriadis T, Pissas G, Liakopoulos V and Stefanidis I: Factors that may protect the native hibernator syrian hamster renal tubular epithelial cells from ferroptosis due to warm anoxia-reoxygenation. Biology (Basel) 8: 22, 2019.
7. Linkermann A, Skouta R, Himmerkus N, Mulay SR, Dewitz C, De Zen F, Prokai A, Zuchtriegel G, Krombach F, Welz PS, et al: Synchronized renal tubular cell death involves ferroptosis. Proc Natl Acad Sci USA 111: 16836-16841, 2014

8. Galluzzi L, Bravo-San Pedro JM, Vitale I, Aaronson SA, Abrams JM, Adam D, Alnemri ES, Altucci L, Andrews D, Annicchiarico-Petruzzelli M, et al: Essential versus accessory aspects of cell death: Recommendations of the NCCD 2015. Cell Death Differ 22: 58-73, 2015.

9. Hrycay EG and Bandiera SM: Monooxygenase, peroxidase and peroxygenase properties and reaction mechanisms of cytochrome P450 enzymes. 851: 1-61, 2015.

10. Lewis DFV: Oxidative stress: The role of cytochromes P450 in oxygen activation. J Chem Technol Biotechnol 77: 1095-1100, 2002.

11. Zeng Q, Han Y, Bao Y, Li W, Li X, Shen X, Wang X, Yao F, O'Rourke ST and Sun C: 20-HETE increases NADPH oxidase-derived ROS production and stimulates the L-type $\mathrm{Ca} 2+$ channel via a PKC-dependent mechanism in cardiomyocytes. Am J Physiol Heart Circ Physiol 299: H1109-H1117, 2010.

12. Han Y, Zhao H, Tang H, Li X, Tan J, Zeng Q and Sun C: 20-Hydroxyeicosatetraenoic acid mediates isolated heart ischemia/reperfusion injury by increasing NADPH oxidase-derived reactive oxygen species production. Circ J 77: 1807-1816, 2013

13. Granville DJ, Tashakkor B, Takeuchi C, Gustafsson AB, Huang C, Sayen MR, Wentworth P Jr, Yeager M and Gottlieb RA: Reduction of ischemia and reperfusion-induced myocardial damage by cytochrome P450 inhibitors. Proc Natl Acad Sci USA 101: 1321-1326, 2004.

14. Ishihara Y, Sekine M, Nakazawa M and Shimamoto N: Suppression of myocardial ischemia-reperfusion injury by inhibitors of cytochrome P450 in rats. Eur J Pharmacol 611: 64-71, 2009.

15. Ishihara Y, Sekine M, Hamaguchi A, Kobayashi Y, Harayama T, Nakazawa M and Shimamoto N: Effects of sulfaphenazole derivatives on cardiac ischemia-reperfusion injury: Association of cytochrome P450 activity and infarct size. J Pharmacol Sci 113: 335-342, 2010.

16. Shaik IH and Mehvar R: Cytochrome P450 induction by phenobarbital exacerbates warm hepatic ischemia-reperfusion injury in rat livers. Free Radic Res 44: 441-453, 2010.

17. Shaik IH and Mehvar R: Effects of cytochrome p450 inhibition by cimetidine on the warm hepatic ischemia-reperfusion injury in rats. J Surg Res 159: 680-688, 2010.

18. Nebert DW, Dalton TP, Okey AB and Gonzalez FJ: Role of aryl hydrocarbon receptor-mediated induction of the CYP1 enzymes in environmental toxicity and cancer. J Biol Chem 279: 23847-23850, 2004.

19. Lindsey $S$ and Papoutsakis ET: The evolving role of the aryl hydrocarbon receptor (AHR) in the normophysiology of hematopoiesis. Stem Cell Rev Rep 8: 1223-1235, 2012.

20. Pollenz RS: The mechanism of AH receptor protein down-regulation (degradation) and its impact on $\mathrm{AH}$ receptor-mediated gene regulation. Chem Biol Interact 141: 41-61, 2002.

21. Ma Q and Baldwin KT: 2,3,7,8-tetrachlorodibenzo-p-dioxininduced degradation of aryl hydrocarbon receptor $(\mathrm{AhR})$ by the ubiquitin-proteasome pathway. Role of the transcription activaton and DNA binding of AhR. J Biol Chem 275: 8432-8438, 2000.

22. Forrester AR, Elias MS, Woodward EL, Graham M, Williams FM and Reynolds NJ: Induction of a chloracne phenotype in an epidermal equivalent model by 2,3,7,8-tetrachlorodibenzo-p-dioxin (TCDD) is dependent on aryl hydrocarbon receptor activation and is not reproduced by aryl hydrocarbon receptor knock down. J Dermatol Sci 73: 10-22, 2014.

23. Yang SC, Wu CH, Tu YK, Huang SY and Chou PC: Exposure to 2,3,7,8-tetrachlorodibenzo-p-dioxin increases the activation of aryl hydrocarbon receptor and is associated with the aggressiveness of osteosarcoma MG-63 osteoblast-like cells. Oncol Lett 16: 3849-3857, 2018.

24. Moretti S, Nucci N, Menicali E, Morelli S, Bini V, Colella R, Mandarano M, Sidoni A and Puxeddu E: The Aryl Hydrocarbon Receptor Is Expressed in Thyroid Carcinoma and Appears to Mediate Epithelial-Mesenchymal-Transition. Cancers (Basel) 12: $145,2020$.

25. Couroucli XI, Liang YW, Jiang W, Barrios R and Moorthy B: Attenuation of oxygen-induced abnormal lung maturation in rats by retinoic acid: Possible role of cytochrome P4501A enzymes. J Pharmacol Exp Ther 317: 946-954, 2006. 
26. Kopf PG, Scott JA, Agbor LN, Boberg JR, Elased KM, Huwe JK and Walker MK: Cytochrome P4501A1 is required for vascular dysfunction and hypertension induced by 2,3,7,8-tetrachlorodibenzop-dioxin. Toxicol Sci 117: 537-546, 2010.

27. Kopf PG and Walker MK: 2,3,7,8-tetrachlorodibenzo-p-dioxin increases reactive oxygen species production in human endothelial cells via induction of cytochrome P4501A1. Toxicol Appl Pharmacol 245: 91-99, 2010.

28. Lee HJ, Pyo MC, Shin HS, Ryu D and Lee K-W: Renal toxicity through AhR, PXR, and Nrf2 signaling pathway activation of ochratoxin A-induced oxidative stress in kidney cells. Food Chem Toxicol 122: 59-68, 2018.

29. Pei J, Juni R, Harakalova M, Duncker DJ, Asselbergs FW, Koolwijk P, Hinsbergh VV, Verhaar MC, Mokry M and Cheng C: Indoxyl sulfate stimulates angiogenesis by regulating reactive oxygen species production via CYP1B1. Toxins (Basel) 11: 454, 2019.

30. Furue M, Takahara M, Nakahara T and Uchi H: Role of AhR/ARNT system in skin homeostasis. Arch Dermatol Res 306: 769-779, 2014.

31. Yagishita Y, Uruno A and Yamamoto M: NRF2-mediated gene regulation and glucose homeostasis. In: Molecular nutrition and diabetes. Elsevier Inc., pp331-348, 2016.

32. Dietrich C: Antioxidant functions of the aryl hydrocarbon receptor. Stem Cells Int 2016: 7943495, 2016.

33. Ma Q: Role of nrf2 in oxidative stress and toxicity. Annu Rev Pharmacol Toxicol 53: 401-426, 2013.

34. Corsello T, Komaravelli $\mathrm{N}$ and Casola A: Role of hydrogen sulfide in NRF2- and sirtuin-dependent maintenance of cellular redox balance. Antioxidants 7: 129, 2018

35. Cuartero MI, Ballesteros I, de la Parra J, Harkin AL, Abautret-Daly A, Sherwin E, Fernández-Salguero P, Corbí AL, Lizasoain I and Moro MA: L-kynurenine/aryl hydrocarbon receptor pathway mediates brain damage after experimental stroke. Circulation 130: 2040-2051, 2014

36. Kaluz S, Kaluzová $M$ and Stanbridge EJ: Regulation of gene expression by hypoxia: Integration of the HIF-transduced hypoxic signal at the hypoxia-responsive element. Clin Chim Acta 395: 6-13, 2008.

37. Myllyharju J: Prolyl 4-hydroxylases, master regulators of the hypoxia response. Acta Physiol (Oxf) 208: 148-165, 2013.

38. Vorrink SU,Sarsour EH, Olivier AK, Robertson LW, Goswami PC and Domann FE: PCB 126 perturbs hypoxia-induced HIF-1a activity and glucose consumption in human HepG2 cells. Exp Toxicol Pathol 66: 377-382, 2014.

39. Vorrink SU, Severson PL, Kulak MV, Futscher BW and Domann FE. Hypoxia perturbs aryl hydrocarbon receptor signaling and CYP1A1 expression induced by PCB 126 in human skin and liver-derived cell lines. Toxicol Appl Pharmacol 274: 408-416, 2014.

40. Eleftheriadis T, Pissas G, Antoniadi G, Liakopoulos V and Stefanidis I: Kynurenine, by activating aryl hydrocarbon receptor, decreases erythropoietin and increases hepcidin production in HepG2 cells: A new mechanism for anemia of inflammation. Exp Hematol 44: 60-7.e1, 2016.
41. Kim SH, Henry EC, Kim DK, Kim YH, Shin KJ, Han MS, Lee TG, Kang JK, Gasiewicz TA, Ryu SH, et al: Novel compound 2-methyl-2H-pyrazole-3-carboxylic acid (2-methyl-4-o-tolylaz o-phenyl)-amide (CH-223191) prevents 2,3,7,8-TCDD-induced toxicity by antagonizing the aryl hydrocarbon receptor. Mol Pharmacol 69: 1871-1878, 2006.

42. Dhillon S: Roxadustat: First Global Approval. Drugs 79: 563-572, 2019.

43. Stockwell BR, Friedmann Angeli JP, Bayir H, Bush AI, Conrad M, Dixon SJ, Fulda S, Gascón S, Hatzios SK, Kagan VE, et al: Ferroptosis: A regulated cell death nexus linking metabolism, redox biology, and disease. Cell 171: 273-285, 2017.

44. Rzemieniec J, Wnuk A, Lasoń W, Bilecki W and Kajta M: The neuroprotective action of 3,3'-diindolylmethane against ischemia involves an inhibition of apoptosis and autophagy that depends on HDAC and AhR/CYP1A1 but not ER $\alpha / C Y P 19 A 1$ signaling. Apoptosis 24: 435-452, 2019.

45. Stejskalova L, Dvorak Z and Pavek P: Endogenous and exogenous ligands of aryl hydrocarbon receptor: Current state of art. Curr Drug Metab 12: 198-212, 2011.

46. Wu PY, Chuang PY, Chang GD, Chan YY, Tsai TC, Wang BJ, Lin KH, Hsu WM, Liao YF and Lee H: Novel endogenous ligands of aryl hydrocarbon receptor mediate neural development and differentiation of neuroblastoma. ACS Chem Neurosci 10: 4031-4042, 2019.

47. Fadeel B and Orrenius S: Apoptosis: A basic biological phenomenon with wide-ranging implications in human disease. J Intern Med 258: 479-517, 2005.

48. Huang B, Bao J, Cao YR, Gao HF and Jin Y: Cytochrome P450 1A1 (CYP1A1) catalyzes lipid peroxidation of oleic acid-induced HepG2 cells. Biochemistry (Mosc) 83: 595-602, 2018.

49. Mohib K, Wang S, Guan Q, Mellor AL, Sun H, Du C and Jevnikar AM: Indoleamine 2,3-dioxygenase expression promotes renal ischemia-reperfusion injury. Am J Physiol Renal Physiol 295: F226-F234, 2008.

50. Fang YZ, Yang S and Wu G: Free radicals, antioxidants, and nutrition. Nutrition 18: 872-879, 2002.

51. Eleftheriadis T, Pissas G, Nikolaou E, Liakopoulos V and Stefanidis I: The H2S-Nrf2-antioxidant proteins axis protects renal tubular epithelial cells of the native hibernator syrian hamster from reoxygenation-induced cell death. Biology (Basel) 8: 74, 2019.

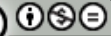

This work is licensed under a Creative Commons Attribution-NonCommercial-NoDerivatives 4.0 International (CC BY-NC-ND 4.0) License. 